\title{
Wake-up radio enabled BLE wearables: empirical and analytical evaluation of energy efficiency
}

\author{
Konstantin Mikhaylov \\ Centre for Wireless Communications \\ University of Oulu \\ Oulu, Finland \\ ORCID: 0000-0002-5518-6629
}

\author{
Heikki Karvonen \\ Centre for Wireless Communications \\ University of Oulu \\ Oulu, Finland \\ ORCID: 0000-0002-1491-0422
}

\begin{abstract}
The energy efficiency is an important issue for a vast range of the Internet of Things (IoT) applications. However, especially critical is the energy efficiency in the context of wearable and body-area network devices. At the very same time, due to their nature, these use cases often impose stringent latency and reliability requirements. In this study, we provide an insight into the feasibility and the performance of the Bluetooth Low Energy (BLE) 5.0 compatible sensor devices enriched with a wake-up radio (WUR). Introduction of a low-consuming WUR radio equips a sensing device with a mechanism to know when its data are desired, and helps to avoid the unnecessary data transmissions thus saving energy. To investigate the utility of this approach and the associated with it trade-offs, we start by instrumenting and measuring a real-life prototype of a WURequipped BLE device. Based on the results of the real-life measurements, we first develop the analytical models, and then analyze how the latency requirements imposed by the application and the number of the wake-up signals affect the energy consumption of the WUR-equipped BLE device and a standalone BLE sensor. Our results show under which conditions each of these architectures outperforms another one and demonstrate that the WUR approach can be more energy efficient in the case if the desired latency for data delivery is below $2.11 \mathrm{~s}$.
\end{abstract}

Index Terms-Wake-up radio, Bluetooth Low Energy, energy efficiency, measurement, experiment, trade-offs

\section{INTRODUCTION}

The world rapidly develops into a heterogeneous interconnected society composing both humans and machines. Over the past few years, myriads of autonomous machines both tiny and huge, simple and complex - have taken their place around us. In 2019 the number of these connected machines topped 26 billion units worldwide [1], exceeding the number of human users several times. Despite the indisputable benefit they bring and the wide variety of the innovative services enabled by these machines, the concerns regarding how efficiently do they operate and use their available resources do arise. The attention to this aspect of technological development has been emphasized by the UN, which has formulated the 17 sustainable development goals [2].

Already today, the ICT sector is estimated to cause $3.6 \%$ of global energy consumption and $1.4 \%$ of $\mathrm{CO} 2$ emissions [3]. With the further development of the 5 and $6 \mathrm{G}$ connectivity [4], as well as due to the increase of the number of the Internetof-Things (IoT) devices, this growing trend is expected to continue. Meanwhile, along with the noticeable negative en- vironmental impact, the increased energy consumption of the IoT devices often compromises their functionality. This is especially notable for the mobile and nomadic IoT devices, such as the wide variety of wearable sensors, actuators and other "human-companion" devices.

Specifically, the conventional mechanism to save the energy for IoT devices is based on the extensive use of low-power sleep modes in which the most energy-hungry functions of the devices, including the ones related to radio connectivity, are disabled. This makes the IoT device temporarily unreachable to the network. Therefore, as a compromise, the contemporary IoT devices often implement duty cycling by periodically switching between a sleep and an active mode. This works well in case of a strictly periodic traffic, however in case of event-based data generation, which are characteristic to some bio-medical applications, this results in consumption of excessive energy or intolerable latency.

However, recently the concept of a wake-up radio has been proposed to address this situation [5]-[8]. It implies integration into an IoT device of a simple and low-consuming radio receiver, which activates the full-functional primary radio system on the reception of a predefined radio signal. The transmitter of this "wake-up" signal, which is usually a less restricted device, might be integrated with the communication infrastructure or deployed as an independent system element. Compared to conventional duty-cycling, the use of a wakeup radio can enable to reduce the idle listening and communication latency at the cost of increasing system cost and complexity, and a minor increase of a sleep mode consumption for sensor. Another advantage of the wake-up radio usage is the reduction of interference by using communication only when necessary for the application. Mitigation against the coexistence problem is important especially in healthcare scenarios to maintain reliability of communication [9].

In the current paper (the major contribution), we systematically study the energy utility of using a wake-up radio in the context of wearable applications. Specifically, we start by prototyping and measuring the key performance characteristics of a device integrating a Bluetooth Low Energy (BLE) 5.0-ready system-on-chip (SoC) and a wake-up radio. We further use the results of the real-life experimental measurements to capture and analyze the key trade-offs, and to specify the scenarios 


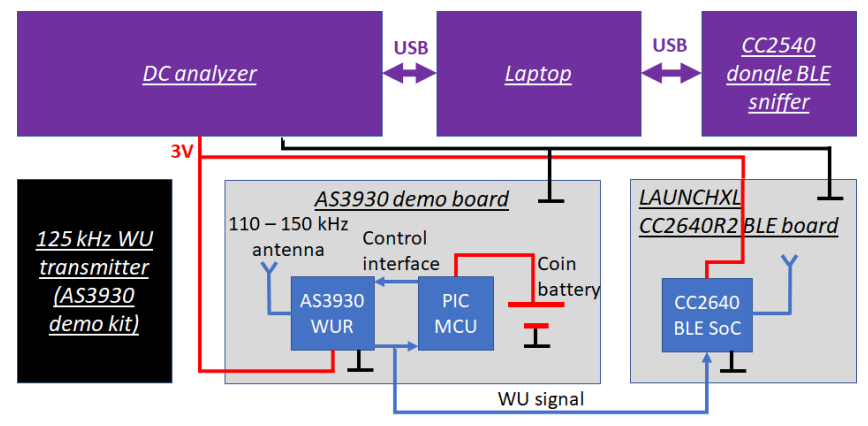

Fig. 1. The structural diagram of the testbed

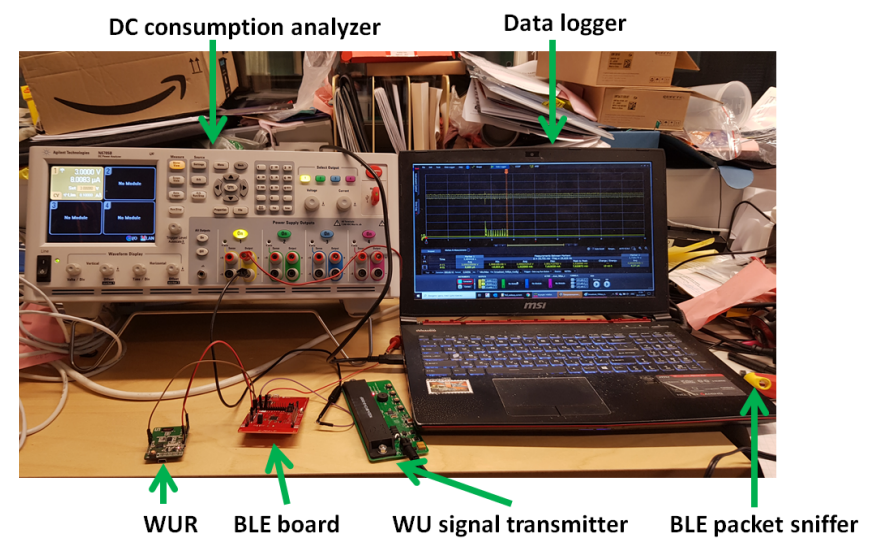

Fig. 2. The photo of the testbed with key components highlighted

and traffic patterns, for which the WUR-based approach outperforms the duty-cycling, and estimate the respective energy consumption and lifetime improvements.

The paper is organized as follows. In Section II, we first explain our experimental setup and then present the results of the empirical measurements. In Section III we start by introducing the energy consumption models for BLE-only and BLE-WUR device and the respective parameters, and then present the results of comparison of energy efficiency of the two architectures as a function of application-layer latency and the number of wake up requests. Finally, Section IV concludes the paper, summarizes and speculates on the obtained results.

\section{EMPIRICAL MEASUREMENTS}

To prove the feasibility and estimate the utility of a WURenabled wearable IoT device, we have prototyped it using the state-of-the-art commercial equipment. The components to be used were selected due to their commercial availability, cost, low energy consumption profile and tiny form-factor, all of which is critical for a wearable application. Note, that we imply the wake-up signal transmitter device to be more resource-rich and thus do not account for its consumption.

\section{A. Experimental setup}

The structural diagram and the photo of the testbed are presented in Figs. 1 and 2, respectively. The testbed is built around the two development boards: the AS3930 WUR demo system from AMS [10] and the CC2640R2 LaunchPad from Texas Instruments [11].
The AS3930 demo kit is composed of (i) the demo board integrating an AS3930 WUR chip [12] with antenna and a microcontroller to control the parameters of WUR and signalize the wake-up, and (ii) a proprietary wake-up signal transmitter for $125 \mathrm{kHz}$ carrier frequency. The wake-up transmitter can be configured to send the wake-up patterns periodically or once a button is pressed. The latter option was used in our experiments. The default WUR configuration, namely the symbol rate of $4096 \mathrm{symbols} / \mathrm{s}$, preamble length of $2.3 \mathrm{~ms}$ and no low power mode, were employed.

The wake-up signal output of the WUR has been connected to one of the input pins of a CC2640R2 LaunchPad development kit. Based on the "simple_broadcaster" software example of the software studio a new firmware version was developed. After startup, the system-on-chip entered the shutdown mode (the low-power mode with lowest consumption possible and no memory retention) and awaited for a wakeup signal. Once receiving it, the CC2640 initialized the BLE stack and started broadcasting the advertisements with a constant component of advertisement period equal to $100 \mathrm{~ms}$ in the three default primary advertising channels. The CC2640 was forced to use the $1 \mathrm{Mbit} / \mathrm{s}$ BLE physical layer, the transmit power was set to $0 \mathrm{dBm}$, and the size of the applicationlevel payload was eight bytes. After exactly one second, the advertisements were disabled, and the BLE radio returned to shutdown mode waiting for the next wake-up.

To measure the current consumption of the deployed system, we have used the Agilent N6705B DC power analyzer connected to the supply lines of the WUR and the C2640R2 LaunchPad. Note that the microcontroller of the AS3930 WUR board and the programmer microcontroller of the LaunchPad board were disconnected from these supply lines and powered from external sources (i.e., a battery and via USB, respectively). To monitor the BLE transmissions by CC2640, we have used a BLE USB dongle together with the packet sniffer software from Texas Instruments, which was configured to filter out the packets sent by the device under test from the background traffic. Note that there was a number of other BLE transmitters in the office environment where the experiments were made, which may have interfered with our system and may result in a few packets getting lost. Also, due to limited range and support for multiple unique codes, the use of WUR does not compromises system scalability.

\section{B. Measurement results}

First of all, we validated the correctness of the resulting system's operation and characterized its critical performance metrics. Specifically, based on our tests, the maximum distance between the wake-up transmitter and the WUR for reliable wake-up (with the probability of over 95\%) was about three meters. At the same time, no false wake-ups have been observed during the experiments.

Next, we placed the WUR and the transmitter at a distance of about one meter and measured the current consumption profiles of our designed system. The results for one of these measurements with clarifications, and captured by the packet 


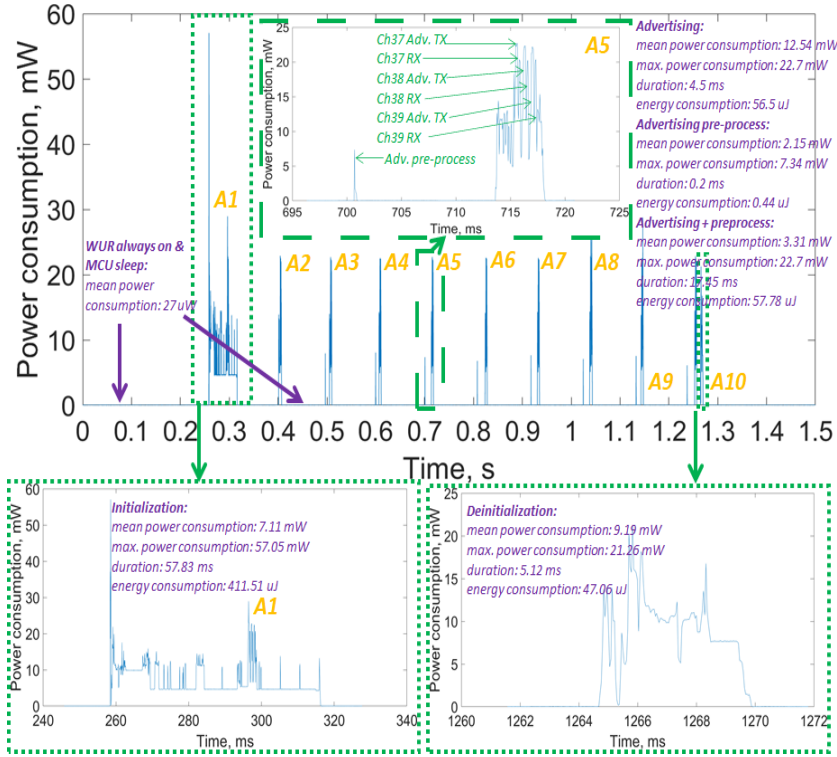

Fig. 3. Power consumption profile of the BLE+WUR system's operation

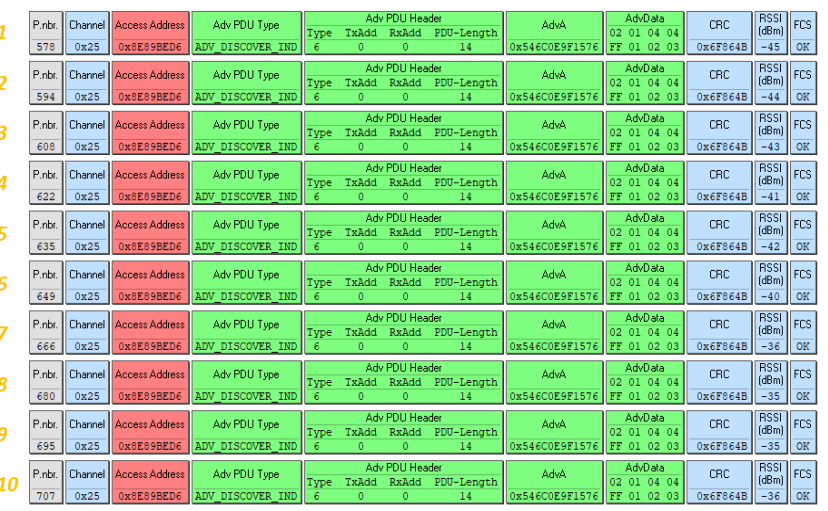

Fig. 4. Packets received by the packet sniffer from the CC2640 system-onchip for the experiment depicted in Fig. 3

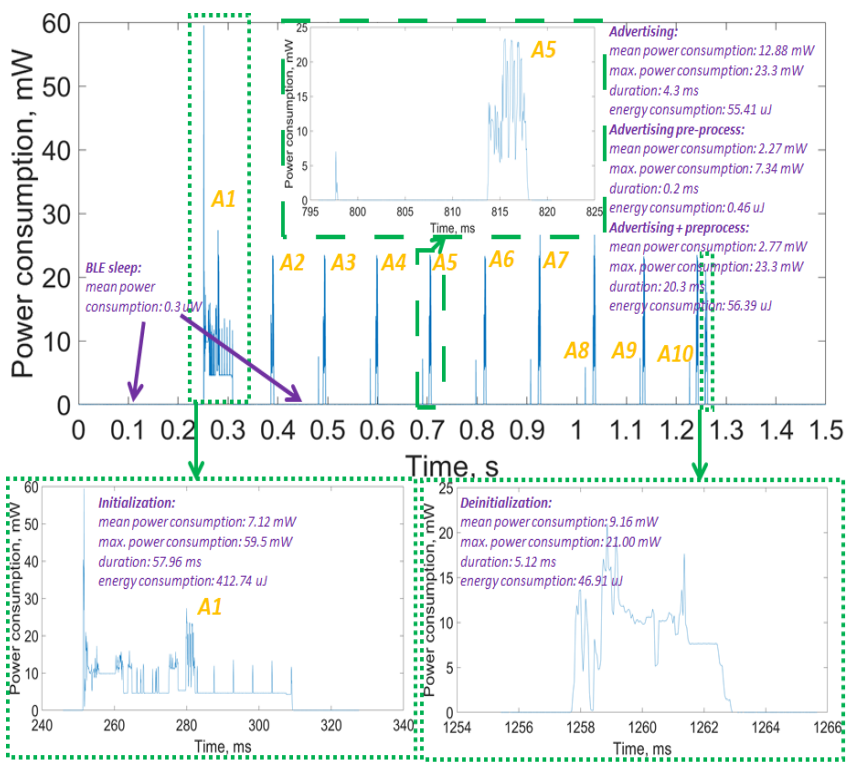

Fig. 5. Power consumption profile of the BLE-only device
TABLE I

NOTATIONS AND PARAMETER VALUES.

\begin{tabular}{|c|c|c|}
\hline Symbol & Definition & Value \\
\hline$P_{\text {Init }}$ & Avg. power consumption of Init-phase & $7.11 \mathrm{~mW}$ \\
$T_{\text {Init }}$ & Duration of Init-phase & $57.83 \mathrm{~ms}$ \\
$P_{\text {Prepro }}$ & Avg. power consumption of & $2.15 \mathrm{~mW}$ \\
& Prepro-phase & \\
$T_{\text {Prepro }}$ & Duration of Prepro-phase & $0.2 \mathrm{~ms}$ \\
$P_{\text {AdvTx }}$ & Avg. power consumption of & $12.54 \mathrm{~mW}$ \\
& AdvTx-phase & \\
$T_{\text {AdvTx }}$ & Duration of AdvTx-phase \\
$P_{\text {Deinit }}$ & Avg. power consumption of & $4.3 \mathrm{~ms}$ \\
& Deinit-phase & $9.19 \mathrm{~mW}$ \\
$T_{\text {Deinit }}$ & Duration of Deinit-phase & $5.12 \mathrm{~ms}$ \\
$P_{W U R \text {,sleep }}$ & Avg. power consumption of WUR & $27 \mu \mathrm{W}$ \\
$T_{W U R, \text { sleep }}$ & sleep-phase & \\
$P_{B L E, \text { sleep }}$ & Duration of WUR sleep-phase & varying \\
$T_{B L E, \text { sleep }}$ & Avg. power consumption of BLE & $0.3 \mu \mathrm{W}$ \\
& Duration of BLE sleep-phase & varying \\
\hline
\end{tabular}

sniffer BLE packets are depicted in Figs. 3 and 4, respectively. The current consumption was sampled at approximately 24.4 ksps rate under $3 \mathrm{~V}$ stable voltage supply, logged, and postprocessed in Matlab environment.

On Fig. 3, one can see all the key phases of the designed system's operation. Prior to receiving a wake-up signal, the BLE radio is off, and the WUR is in listening state, resulting in the average power consumption of around $27 \mu \mathrm{W}$. After getting the wake-up signal, the BLE SoC initializes and sends the first broadcast packet. Once initialization is completed, the main processing core of the $\mathrm{SoC}$ goes to sleep mode, and the separate BLE radio controller takes care about sending the subsequent advertisements. One can notice that the advertisement transmissions are not regular, which is caused by the presence of an up-to $10 \mathrm{~ms}$ long pseudo-random delay component. One second after the start, the $\mathrm{SoC}$ deactivates the radio and goes to the low power mode.

The similar measurements have been conducted by us for the case when no WUR was connected, and a button was used to wake the $\mathrm{SoC}$ up. The respective results are depicted in Fig. 5. The major difference between the two scenarios, as can be seen, is the sleep-mode consumption. For the BLE-only case, it went down to a mere $0.3 \mu \mathrm{W}$.

\section{ANALYTICAL EVALUATION}

In this section, we first introduce the analytical model for energy consumption comparison which we use to study and compare the consumption of wake-up radio enabled against the baseline (i.e., a duty-cycling based BLE-only) scenario, and then present the respective results. The presented model provides a more comprehensive picture concerning the performance comparison in addition to measurement results done for a single wake-up cycle. Note that the analytical modelling is based on the real measurement results, which were introduced in the previous section. Thus, the presented results provide a realistic view of energy consumption comparison of sensor.

Our measurements have identified the different phases of the radio devices' operation, and the corresponding power consumption values, during the sleep, wake-up and broadcast 
periods. In what follows, we specify the notations used in our analytical model based on the identified actions. For both scenarios considered, there is sleep phase average power consumption which is defined to be $P_{R \text {,sleep }}$, where $R$ is WUR $\&$ BLE, or BLE-only radio, for the WUR-enabled and the baseline scenarios, respectively. Note, that the WUR listens to the channel and can awake the main radio anytime, while BLE-only radio does not know when the receiver is near and/or needs the data. The sleep period duration is defined to be $T_{R \text {,sleep }}$, which can be different, depending on whether this is a WUR+BLE or a BLE-only radio.

In the case of a WUR \& BLE radio, after receiving the wake-up signal, it operates in three phases: Initialization (Init), Advertising (Adv) and Deinitialization (Deinit). Furthermore, the Adv-phase is composed of the two sub-phases: Preprocessing (Prepro) and Advertising Transmissions (AdvTx). Note, that to increase the probability of data delivery, the advertisements can be repeated multiple $(N)$ times.

In the case of a BLE-only radio, we consider that initialization is done only once in the very beginning of operation (thus this consumption is not considered). Then the radio periodically broadcasts the advertisements (the broadcast data may be periodically changed based, e.g., on the data from sensors). Therefore, except for the sleep mode, the operation includes only the Adv phase.

Table I introduces the notations used and parameter values. Next, we define as a single cycle the time between the two wake-ups for WUR \& BLE radio, or between the two BLE advertisements for BLE-only radio. The energy consumption for a single cycle is thus given by:

$$
E_{R, \text { Cycle }}=E_{R, \text { sleep }}+E_{\text {Init }}+N\left(E_{\text {Prepro }}+E_{\text {AdvTx }}\right)+E_{\text {Deinit }},
$$

including energy consumption of the Sleep, Initialization (for WUR \& BLE radio only), Preprocessing, Advertising Transmissions and Deinitialization (for WUR \& BLE radio only) phases, which can be calculated using the average power consumption and time duration parameters of the respective phases (by simply multiplying these) given in Table I. Note that the sleep-phase also includes the silent periods (that can be observed from the power consumption profile presented in the previous section) between different actions.

This can be easily seen that for equal $T_{R \text {,sleep values, the }}$ consumption for WUR \& BLE case is significantly higher than that for BLE-only scenario. However, since the BLE-only radio typically has no idea when a receiver may need the data, it will have to transmit the data periodically. Notably, the more stringent latency requirements the application imposes, the more often the BLE radio will have to repeat its transmission. To give a practical example, if the data should be delivered within a period $l=1 s$, the duty cycle $D C$ of the transmission cannot go below $\left(T_{\text {Prepro }}+T_{\text {AdvTx }}\right) / l=4.5 \mathrm{~ms} / 1 \mathrm{~s}=0.45 \%$.

Next, we consider a time period of $T$ during which a receiver(s) require(s) the data exactly $W$ times, and the maximum possible application latency for data delivery is $l$. As easy to see, under these assumptions for WUR \& BLE radio, the average cycle time does not depend on $l$ and is

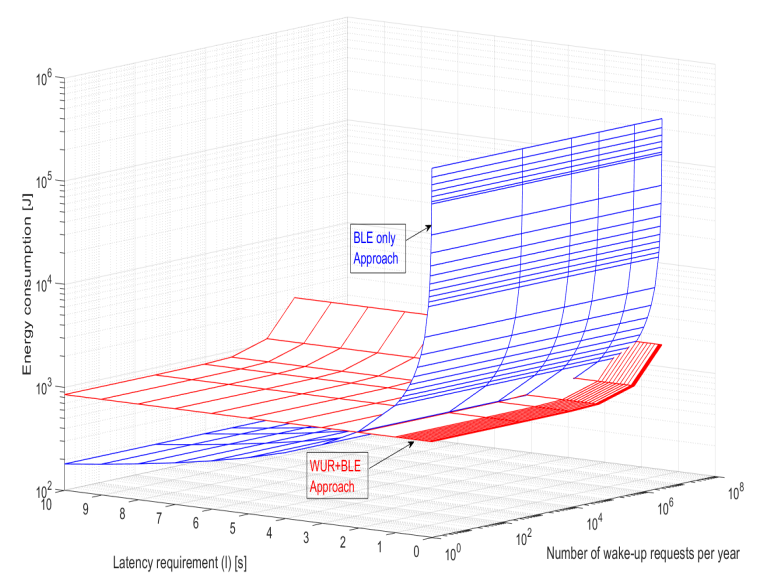

Fig. 6. Energy consumption comparison as a function of duty cycle percentage and number of wake-up request

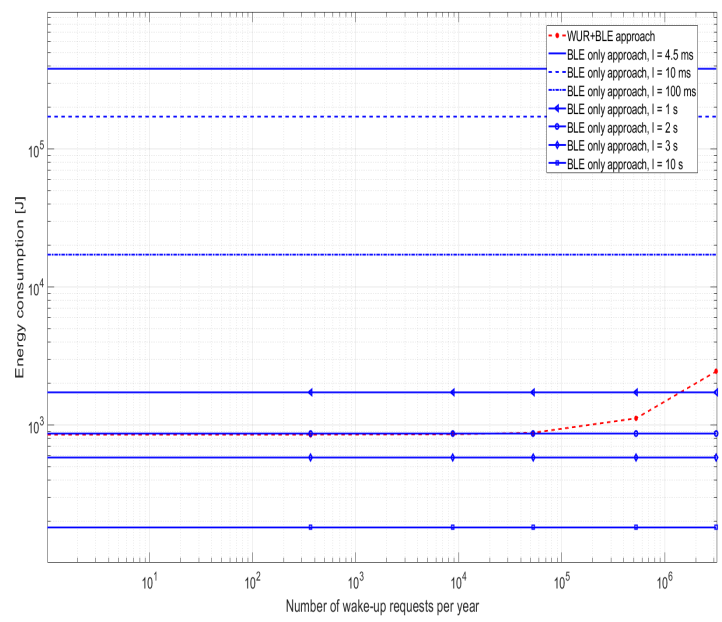

Fig. 7. Energy consumption comparison as a function of number of wake-up request for different duty cycle value cases

given by $T_{R, \text { Cycle }}=T / W$. Meanwhile for the BLE-only radio the average cycle time equals $l$, which related to the DC as $D C=\left(T_{\text {Prepro }}+T_{\text {AdvTx }}\right) / l$. Note, that here we do not account for the potential packet losses.

Based on the discussed models and parameters, Fig. 6 provides an illustrative comparison of the energy consumption of BLE-only and WUR \& BLE radios as a function of the number of events and the maximum latency for a one-year time period. From the figure can be observed the trend that the energy consumption of BLE-only radio increases drastically with the reduction of the maximum allowed latency. Similarly, with the increase in the number of data requests, the consumption of the WUR \& BLE radio goes up. Considering the extremes, in case if a decently high latency is possible, the BLE-only radio will outperform a WUR \& BLE radio with respect to energy consumption one order of magnitude. Specifically, in the case if the average consumption of BLEonly system becomes lower than that of a WUR \& BLE radio during sleep, making BLE-only system always more energy 
efficient. However, in case when maximum permitted latency is in the order or dozens ms and the low number of events the energy efficiency of a WUR \& BLE radio is up to two orders of magnitude higher than that of a BLE-only radio.

$$
l>\frac{P_{\mathrm{AdvTx}} T_{\mathrm{AdvTx}}-P_{B L E, \text { sleep }} T_{\mathrm{AdvTx}}}{P_{W U R, \text { sleep }}-P_{B L E, \text { sleep }}}=2.113 \mathrm{~s}
$$

Figure 7 shows energy consumption comparison for the selected latency values to illustrate in more details the numerical performance difference. It can be observed that when the tolerable latency is more than $2 \mathrm{~s}$, the BLE-only radio outperforms the WUR \& BLE combination. When the permitted latency is $2 \mathrm{~s}$, the WUR \& BLE combination becomes more efficient energy-wise if the number wake-up request per year is not more than $5 \times 10^{4}\left(T_{R \text {,Cycle }}<630 \mathrm{~s}\right)$. From Fig. 7 can be seen that energy consumption saving provided by WUR-enabled BLE solution can be more than two orders of magnitude if latency requirement is around ten $\mathrm{ms}$.

\section{Discussion AND CONCLUSIONS}

In the paper we have investigated the feasibility, the potential performance limits, and their trade-offs, for a system combining a wake-up radio and a less energy restricted BLE v5.0 compatible radio transceiver. Specifically, first, we instrumented and measured the characteristics of a reallife prototype device. Then, we proposed an analytic sensor energy consumption model and used the measured parameters to investigate under which conditions the WUR \& BLE combination would be more energy-efficient than a BLE-only device, which saves energy using duty cycling.

Among the notable results of the practical implementation phase are (i) the proven feasibility of a practical BLE and WUR combination, (ii) the values of the practical power consumption $(0.3 \mu \mathrm{W}$ for BLE-only sleep, $27 \mu \mathrm{W}$ for WUR $\&$ BLE background consumption, and the results for BLE data transfers). One can see that even though the consumption of a WUR is rather low, it still exceeds the level of a BLEonly system sleep mode by almost two orders of magnitude. Note, that there exist research WUR prototypes with lower consumption - results for these will differ.

Next, we have developed analytical models for BLE and WUR combination and BLE-only radio, which enable to estimate the energy consumption of the system as a function of the number of wake-ups in a period of time and the maximum application-level latency. These models and measurement results have been utilized to investigate for which scenarios each approach is more beneficial. Our analyses show that the combination of WUR \& BLE can outperform the BLE-only solution in the case if the maximum latency for data delivery tolerable by the application does not exceed $2.1 \mathrm{~s}$ and the number of data requests to WUR is decently low. Otherwise, the duty-cycling BLE-only radio would consume less energy.

In our opinion, the results, models, and observations presented in this paper are especially useful in the context of developing the smart wearable and intelligent healthcare applications on the one hand, and the smart human-centric environment on the other. By their very nature, the wearable sensors are limited for their energy. Meanwhile, in the context of smart healthcare applications, the maximum tolerable latency can easily go into sub-second orders. Another potential use case implies the communication between a wearable or a smartphone of a moving human user (or even a car) and a statically deployed sensor (e.g., near to the door of a room or a shop). In the latter scenario, the latency is restricted due to the limited duration of a time period the user spends in the proximity of the sensor.

The two major limitations of the current study are (i) lack of consideration of packet loss for WUR or BLE, (ii) no consideration of potential interference and their effect on system's scalability, especially for the case of BLE-only radios, and (iii) consideration of only a single traffic pattern. Note, that in the case of stringent latency and/or reliability requirements the BLE-only sensors will have to repeat their transmissions very often, thus increasing the probability of their packet collisions as well as introducing additional interferences to the other systems operating in the $2.4 \mathrm{GHz}$ ISM band. In contrast to this, the WUR-enabled systems will send their data only once these are needed and requested. Also we limited our study to specific hardware and used $1 \mathrm{Mbps}$ PHY only. In our future works, we plan to address also these issues.

\section{ACKNOWLEDGEMENT}

This research has been partially financially supported by the Academy of Finland 6Genesis Flagship (grant 318927) and by Business Finland through the SP400 project.

\section{REFERENCES}

[1] Leftronic, IoT statistics and trends to know in 2019, available: https://leftronic.com/internet-of-things-statistics/

[2] UN, Envision2030: 17 goals to transform the world for persons with disabilities, available: https://www.un.org/development/desa/disabilities/envision2030.html

[3] J. Malmodin, and D. Lunden, 'The energy and carbon footprint of the global ICT and E\&M sectors 2010-2015,' Sustainability, vol. 10, 2018, paper 3027.

[4] M. Latva-aho, and K. Leppanen (eds.), Key drivers and research challenges for $6 \mathrm{G}$ ubiquitous wireless intelligence, available: http://urn.fi/urn:isbn:9789526223544

[5] J. Petäjäjärvi et al., "WBAN energy efficiency and dependability improvement utilizing wake-up receiver,' IEICE Trans. on Commun., vol. E98-B, no. 04, pp. 535-542, Apr. 2015.

[6] J. Oller et al., "Has Time Come to Switch From Duty-Cycled MAC Protocols to Wake-Up Radio for Wireless Sensor Networks?,' IEEE/ACM Trans. on Networking, vol. 24, no. 2, pp. 674-687, April 2016.

[7] F. Z. Djiroun and D. Djenouri, "MAC Protocols With Wake-Up Radio for Wireless Sensor Networks: A Review,' IEEE Commun. Surv. \& Tut., vol. 19, no. 1, pp. 587-618, Firstquarter 2017.

[8] H. Karvonen, J. Petäjäjärvi, J. Iinatti, and M. Hämäläinen, ”Energy Efficient IR-UWB WBAN using a Generic Wake-up Radio based MAC Protocol", in Proc. Int. Conf. Body Area Networks, London, UK, Sep 29 - Oct 1, 2014.

[9] H. Karvonen, M. Hämäläinen, J. Iinatti, and C. Pomalaza-Ráez, "Coexistence of wireless technologies in medical scenarios," Eur. Conf. Networks and Commun., Oulu, June 2017.

[10] AMS, AS3930 Standard Board, AS3930 DEMOSYSTEM, Demo Kit Manual v1-02, Jul. 2014.

[11] Texas Instruments, CC2640R2 LaunchPad Quick Start Guide (Rev. B), SWRU500b, 2019.

[12] AMS, AS3930 Single Channel Low Frequency Wakeup Receiver, Datasheet v1-62, Nov. 2014. 